\title{
Ozonización De Residuos Líquidos Textiles Para Remoción De Sólidos Suspendidos Totales, Demandas Química Y Bioquímica De Oxígeno, Grasas Y Aceites
}

\author{
Agustín Leiva Pérez \\ Edison Macías Andrade \\ Teódulo Zambrano Farías
}

Docentes e Investigadores de la escuela Superior Politécnica Agropecuaria

de Manabí "Manuel Félix López"

Roy Barre Zambrano

Docente e Investigador de la Pontificia Universidad Católica del Ecuador

Ricardo Montesdeoca Párraga

Joffre Andrade Candell

Docentes e Investigadores de la escuela Superior Politécnica Agropecuaria de Manabí "Manuel Félix López"

Doi: 10.19044/esj.2018.v14n3p40 URL:http://dx.doi.org/10.19044/esj.2018.v14n3p40

\begin{abstract}
The study of the textile industry liquid wastes oxidation by ozone application in completed mixed batch reactors, with reaction times between 10 and 60 minutes, was developed according to previous observation tests. A SEFILTRA Ozone Generator, manufactured in Madrid, Spain, characterized by an air flow of $2 \mathrm{dm}^{3} / \mathrm{min}$ ), ozone concentration of $9,50 \mathrm{mg} / \mathrm{dm}^{3}$, an $\mathrm{O}_{3}$ production of $19 \mathrm{mg} / \mathrm{min}$ and \%weight of 0,8 . The contamination indicating parameters as Color, Turbidity, Total Suspended Solids, Chemical Oxygen Demand, Biochemical Oxygen Demand and, Fats and oils, showed satisfactory oxidative levels, with $\mathrm{BOD}_{5}$ and Fats and oils being the most oxidized within 50 minutes of reaction time. All contamination indicators were oxidized by ozone, according to second degree polynomial behavior, with correlation coefficients above 0,98 .
\end{abstract}

Keywords: Oxidation, liquid waste from the textile industry, fully mixed batch reactors, Total Suspended Solids, Chemical Oxygen Demand, Biochemical Oxygen Demand and, Fats and Oils 


\section{Resumen}

Se desarrolló el estudio de la oxidación de los residuos líquidos de la industria textil mediante aplicación de ozono en reactores batch completamente mezclados, con tiempos de reacción entre 10 y 60 minutos, según pruebas de observación realizadas anteriormente. Se utilizó un equipo Generador de Ozono SEFILTRA, fabricado en Madrid, España, que se caracteriza por un flujo aire de $2 \mathrm{dm}^{3} / \mathrm{min}$ ), concentración de ozono de 9,50 $\mathrm{mg} / \mathrm{dm}^{3}$, una producción de $\mathrm{O}_{3}$ de $19 \mathrm{mg} / \mathrm{min}$ y un $\%$ peso de 0,8 . Los parámetros indicadores de la contaminación Sólidos Suspendidos Totales, Demanda Química de Oxígeno, Demanda Bioquímica de Oxígeno y, Grasas y aceites, mostraron niveles oxidativos satisfactorios, siendo la $\mathrm{DBO}_{5}$ y las Grasas y aceites los más oxidados a los 50 minutos de reacción. Todos los parámetros indicadores de la contaminación estudiados resultaron oxidados vía ozonización, según comportamientos polinómicos de segundo grado, con coeficientes de correlación por sobre los 0,98.

Palabras-clave: Oxidación, residuos líquidos de la industria textil, reactores batch completamente mezclados, Sólidos Suspendidos Totales, Demanda Química de Oxígeno, Demanda Bioquímica de Oxígeno y, Grasas y aceites

\section{Introducción}

Aprovechando el hecho probado de que el gas ozono $\left(\mathrm{O}_{3}\right)$ constituye un estado alotrópico del oxígeno $\left(\mathrm{O}_{2}\right)$, generado por el paso de la corriente eléctrica a través de un conductor, preferentemente metálico, se han desarrollado y comercializado una gran variedad de equipos productores de ozono, que incluso, combinan líquidos con la corriente de aire (contiene un $20 \%$ de $\mathrm{O}_{2}$ gaseoso) o de oxígeno ozonizada, que provoca una oxidación enérgica de los diferentes tipos de materiales contenidos en dicho líquidos.

Leiva et al. (2016) reportaron la decoloración del licor negro generado en el proceso de producción de pulpa y papel de bagazo de caña, que poseen altos contenidos del polifenol lignina, obteniendo resultados de entre 53 y $94 \%$ de eliminación del color entre 10 y 60 minutos de reacción ozono - colorantes y; entre 46 y 90\% de remoción de Demanda Química de Oxígeno (DQO) al cabo de los mismos intervalos de tiempo, reduciéndose dichas eficiencias en la medida en que aumentaba el nivel de Sólidos Suspendidos Totales (SST) entre 50 y $300 \mathrm{mg} / \mathrm{dm}^{3}$.

De acuerdo De Jesús (2016) y Bolaños (2010), los residuos líquidos de las industrias textiles también se caracterizan por elevadas concentraciones de color, DQO y otros parámetros indicadores de la contaminación de las aguas como Conductividad eléctrica, Carbono Orgánico Total (COT), Agentes Tensoactivos Catiónicos, Nitrógeno, Grasas y Cloruros, entre otros. El ozono 
por sus características de oxidante enérgico es capaz de eliminar el color en todos sus orígenes y tipos, en cambio, otros tratamientos se emplean para sólo determinadas fuentes de este.

El estudio realizado tuvo el objetivo de evaluar la capacidad oxidativa del ozono en la estabilización de residuos líquidos de la industria textil, empleando un equipo generador de ozono e indicadores de la contaminación como los mencionados anteriormente. La oxidación de la materia orgánica por el ozono es tan rápida que, en el costo del proceso, se impone y repercute positivamente en el costo del mismo, con la conveniencia ambiental de la no generación de lodos ni de efluentes con valores de DQO apreciables.

\section{Materiales y métodos}

Se utilizó un equipo generador de Ozono de la firma madrileña SEFILTRA, que utiliza aire como insumo de $\mathrm{O}_{2}$ y cuyas características principales de interés para el estudio se muestran en el Cuadro 1 y que ya fue empleado por Leiva et al. (2016). Asimismo se dispuso de:

- $\quad$ Equipo de filtración común.

- $\quad$ Filtros para análisis gravimétrico: AP40 Millipore.

- Mangueras plásticas de PVC de 0,5 cm de diámetro interior.

- $\quad$ Reactor batch plástico oscuro de forma cilíndrica circular recta, de 8 $\mathrm{dm}^{3}$ de volumen total (1,27 dm de diámetro de la base y $2 \mathrm{dm}$ de altura) con entrada y salida de gases y toma de muestra en el punto central de su altura efectiva (3,2 dm), algo más pequeño que el empleado por Leiva et al. (2016) en la ozonización de licor negro residual de la producción de pulpa y papel de bagazo de caña. Para propiciar la representatividad de la muestra de la población de residuos líquidos a tratar, se mezclaron $30 \mathrm{dm}^{3}$ de los efluentes de cada una de la empresas textiles JHONATEX ubicada en Leonardo Pérez 01-90 y Homero Hidrovo, Ambato, Tungurahua; TEXTIL BUENAÑO, en la calle 4 del Parque industrial de la misma ciudad y; MODATEX, situada en Bolívar y Mariano Cuevas esq. Cuenca, Azuay. En total, $90 \mathrm{dm}^{3}$, a los que se les realizaron tres repeticiones de análisis después de la mezcla (muestras A, B y C) del Cuadro 2; utilizando los valores medios como iniciales del proceso de ozonización. Según los resultados de varias pruebas de observación se seleccionaron tiempos de ozonización de 10, 20, 30, 40, 50 y 60 minutos, repitiéndose tres veces cada proceso, nominados como $\mathrm{P}_{10}, \mathrm{P}_{20}$ y $\mathrm{P}_{30}, \mathrm{P}_{40}, \mathrm{P}_{50}$ y $\mathrm{P}_{60}$,respectivamente.

Para cada tiempo de ozonización el reactor trabajó con $5 \mathrm{dm}^{3}$ de volumen en cada una de las tres réplicas con cada tiempo. De esta forma, en el reactor siempre quedaron $3 \mathrm{dm}^{3}$ de volumen libre para evitar pérdidas por derrames debido a la espuma. En la Figura 1 se muestra un esquema del reactor que fue empleado en los seis procesos batch, almacenándose en frío, el líquido 
que va quedando sin usar hasta el batch en función y que se utilizó después en los restantes.

Cuadro 1. Características principales utilizadas del equipo Generador de Ozono SEFILTRA, Madrid, España.

\begin{tabular}{|c|c|c|c|}
\hline Flujo aire $\left(\mathrm{dm}^{3} / \mathrm{min}\right)$ & $\mathrm{c}\left(\mathrm{O}_{3}\right)\left(\mathrm{mg} / \mathrm{dm}^{3}\right)$ & Producción de $\mathrm{O}_{3}(\mathrm{mg} / \mathrm{min})$ & $\%$ Peso \\
\hline 2 & 9,50 & 19 & 0,8 \\
\hline
\end{tabular}

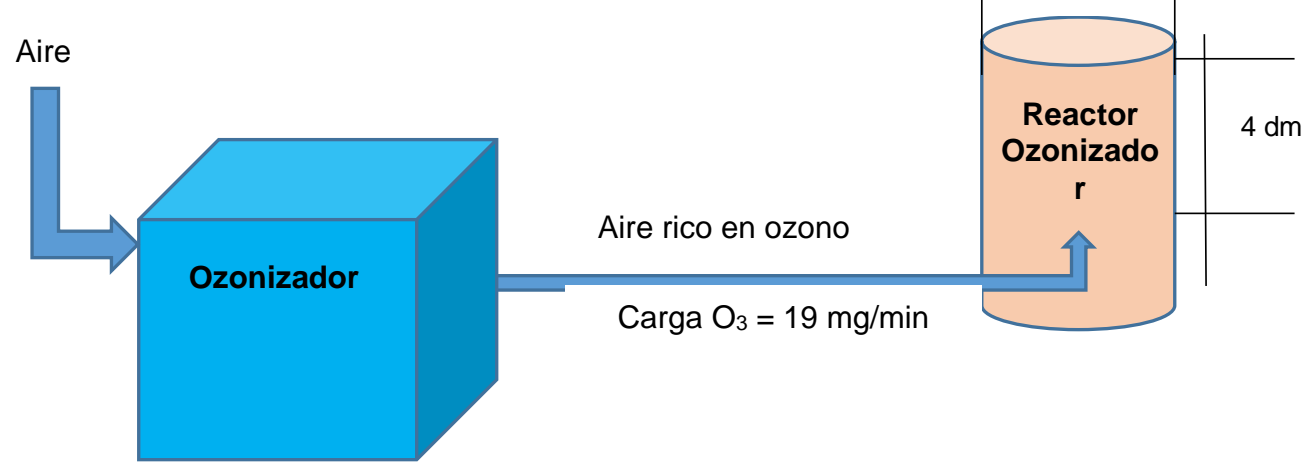

Figura 1. Esquema general del proceso de ozonización.

En el Cuadro 2 se muestran las características principales del residuo líquido textil mezclado utilizado en los tratamientos basados en la ozonización, indicándose sus valores medios, desviaciones estándar y coeficientes de variabilidad.

Cuadro 2. Características principales del agua residual textil mezclada utilizada en los tratamientos basados en la ozonización.

\begin{tabular}{|c|c|c|c|c|c|c|c|}
\hline Parámetro & Unidad & $\begin{array}{c}\text { Muestra } \\
\text { A }\end{array}$ & $\begin{array}{c}\text { Muestra } \\
\text { B }\end{array}$ & $\begin{array}{c}\text { Muestra } \\
\text { C }\end{array}$ & Media & $\begin{array}{c}\text { Desviación } \\
\text { Estándar }\end{array}$ & $\begin{array}{c}\text { Coeficiente } \\
\text { de Variación } \\
(\%)\end{array}$ \\
\hline $\begin{array}{c}\text { Sólidos } \\
\text { Suspendidos } \\
\text { Totales (SST) }\end{array}$ & $\mathrm{mg} / \mathrm{dm}^{3}$ & 198 & 210 & 205 & 204,33 & 6,03 & 2,95 \\
\hline $\begin{array}{c}\text { Demanda } \\
\text { Química de } \\
\text { Oxígeno } \\
\text { (DQO) }\end{array}$ & $\mathrm{mg} / \mathrm{dm}^{3}$ & 1154 & 1263 & 1160 & 1192,33 & 61,27 & 5,14 \\
\hline $\begin{array}{c}\text { Demanda } \\
\text { Bioquímica } \\
\text { de Oxígeno } \\
\text { (DBO5) }\end{array}$ & $\mathrm{mg} / \mathrm{dm}^{3}$ & 752 & 731 & 685 & 722,67 & 34,27 & 4,74 \\
\hline $\begin{array}{c}\text { Grasas y } \\
\text { aceites (G y } \\
\text { A) }\end{array}$ & $\mathrm{mg} / \mathrm{dm}^{3}$ & 13,3 & 13,8 & 12,4 & 13,17 & 0,71 & 5,39 \\
\hline
\end{tabular}

Todos los análisis se realizaron con base en APHA, AWWA, WPCF (Métodos Estándar...), 2005. 


\section{Resultados y discusión}

En los Cuadros 3, 4 y 5 se presentan los resultados de los procesos $\mathrm{P}_{10}$, $\mathrm{P}_{20}, \mathrm{P}_{30}, \mathrm{P}_{40}, \mathrm{P}_{50}$ y $\mathrm{P}_{60}$ promedios de las tres réplicas, con sus correspondientes eficiencias de remoción de los diferentes parámetros indicadores de la contaminación, sus valores medios, desviaciones estándar y coeficientes de variación. En las discusiones siguientes debe observarse que los valores de los coeficientes de correlación que aparecen insertados en los gráficos so cuadráticos, por lo que es necesario la extracción de las raíces cuadradas.|

Cuadro 3. Resultados de procesos con 10 - 60 min de tiempo de ozonización $\left(\mathrm{P}_{10}, \mathrm{P}_{20}, \mathrm{P}_{30}\right.$, $\left.\mathrm{P}_{40}, \mathrm{P}_{50}, \mathrm{P}_{60}\right) .\left(\mathrm{P}_{10}=\right.$ Proceso oxidativo de $10 \mathrm{~min}, \mathrm{P}_{20}=$ Proceso oxidativo de $10 \mathrm{~min}, \mathrm{y}$ así

sucesivamente).

\begin{tabular}{|c|c|c|c|c|}
\hline Tratamiento & $\% \mathrm{R}_{\mathrm{SST}}$ & $\% \mathrm{R}_{\mathrm{DQO}}$ & $\%_{\mathrm{DBO}}$ & R $_{\mathrm{G}} \mathrm{y} \mathrm{A}$ \\
\hline $\mathrm{P}_{10}$ Media & 15,33 & 19,67 & 25,33 & 22,00 \\
\hline $\mathrm{P}_{10}$ D.E. & 1,15 & 1,53 & 1,53 & 2,65 \\
\hline $\mathrm{P}_{10}$ C.V. & 7,53 & 7,77 & 6,03 & 12,03 \\
\hline $\mathrm{P}_{20}$ Media & 27,67 & 34,00 & 33,00 & 32,00 \\
\hline $\mathrm{P}_{20}$ D.E. & 9,38 & 11,66 & 11,73 & 10,01 \\
\hline $\mathrm{P}_{20}$ C.V. & 33,92 & 34,28 & 35,56 & 31,29 \\
\hline $\mathrm{P}_{30}$ Media & 42,33 & 56,33 & 65,33 & 59,00 \\
\hline $\mathrm{P}_{30}$ D.E. & 2,89 & 2,08 & 2,89 & 1,73 \\
\hline $\mathrm{P}_{30}$ C.V. & 6,82 & 3,70 & 4,42 & 2,94 \\
\hline $\mathrm{P}_{40}$ Media & 53,00 & 75,00 & 83,00 & 74,67 \\
\hline $\mathrm{P}_{40}$ D.E. & 2,65 & 3,61 & 1,73 & 2,89 \\
\hline $\mathrm{P}_{40}$ C.V. & 4,99 & 4,81 & 2,09 & 3,87 \\
\hline $\mathrm{P}_{50}$ Media & 61,33 & 83,67 & 90,67 & 91,00 \\
\hline $\mathrm{P}_{50}$ D.E. & 1,15 & 3,51 & 2,08 & 2,00 \\
\hline $\mathrm{P}_{50}$ C.V. & 1,88 & 4,20 & 2,30 & 2,20 \\
\hline $\mathrm{P}_{60}$ Media & 63,00 & 86,33 & 93,00 & 94,33 \\
\hline $\mathrm{P}_{60}$ D.E. & 2,00 & 1,53 & 2,65 & 2,08 \\
\hline $\mathrm{P}_{60}$ C.V. & 3,17 & 1,77 & 2,84 & 2,21 \\
\hline & & &
\end{tabular}

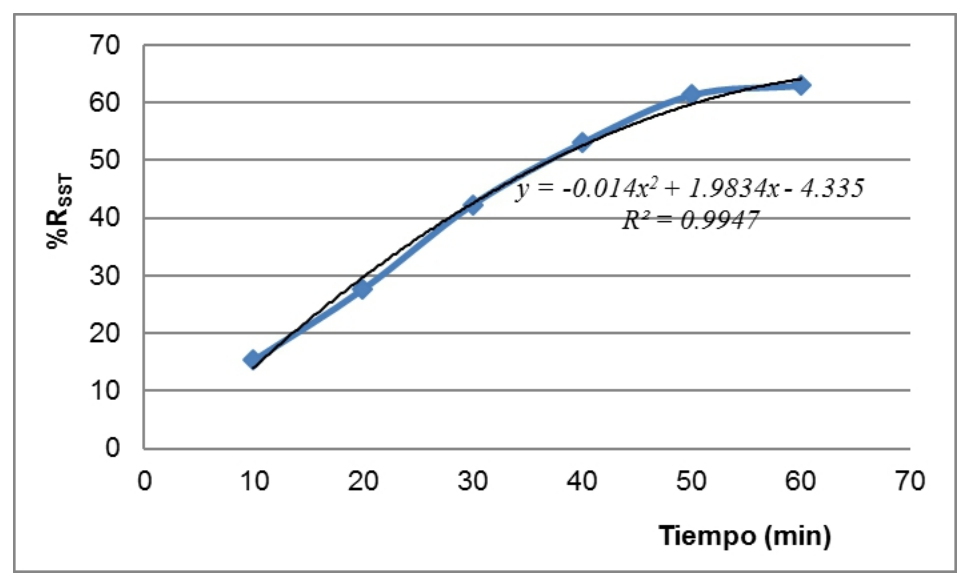

Figura 2. Eficiencia de Remoción de Sólidos Suspendidos Totales 
La eficiencia de eliminación de SST se comportó análogamente que la de los parámetros anteriores, tendiendo a estabilizarse al alcanzar el $60 \%$ de remoción. El comportamiento se correspondió con una ecuación de segundo grado $\left(\mathrm{y}=-0,014 \mathrm{x}^{2}+1,9834 \mathrm{x}-4,335\right)$, ajustándose un coeficiente de correlación de más de 0,99. (Figura 2). En la ozonización de otros residuos líquidos, como los pertenecientes a la industria papelera, los SST producen reducciones en la eficiencia del proceso de decoloración, fenómeno que no se produjo en este tipo acción.

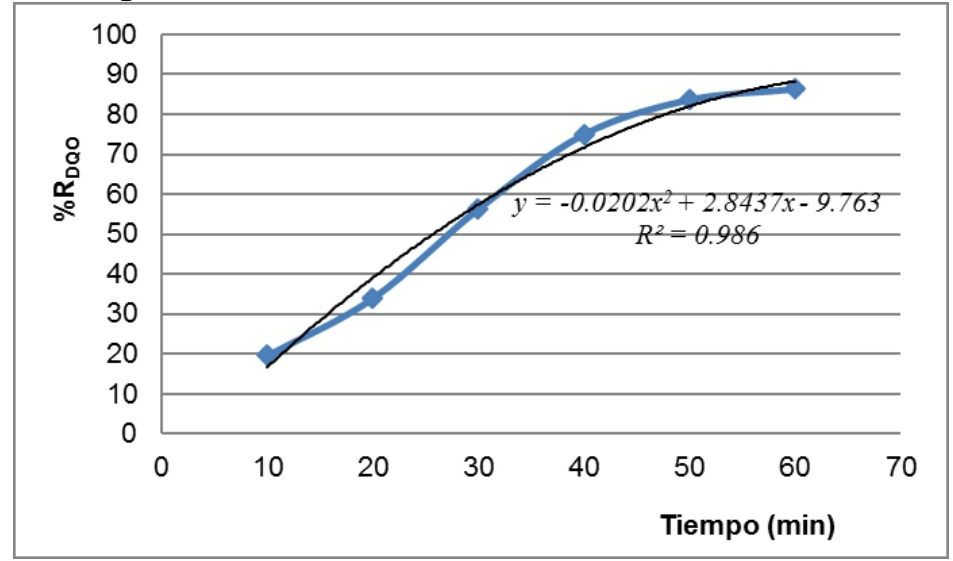

Figura 3. Eficiencia de Remoción de Demanda Química de Oxígeno

En el proceso de oxidación de los compuestos que contribuyen la DQO el ozono actúa de manera eficaz, alcanzándose el $80 \%$ de eliminación al cabo de los 45 min. La tendencia fue alcanzar el $97 \%$ al cabo de la hora de tratamiento. El comportamiento se puede plantear como polinómico de segundo grado $\left(\mathrm{y}=-0,0202 \mathrm{x}^{2}+2,8437 \mathrm{x}-9,763\right)$, en este caso con un coeficiente de correlación de 0,99 (Figura 3 ).

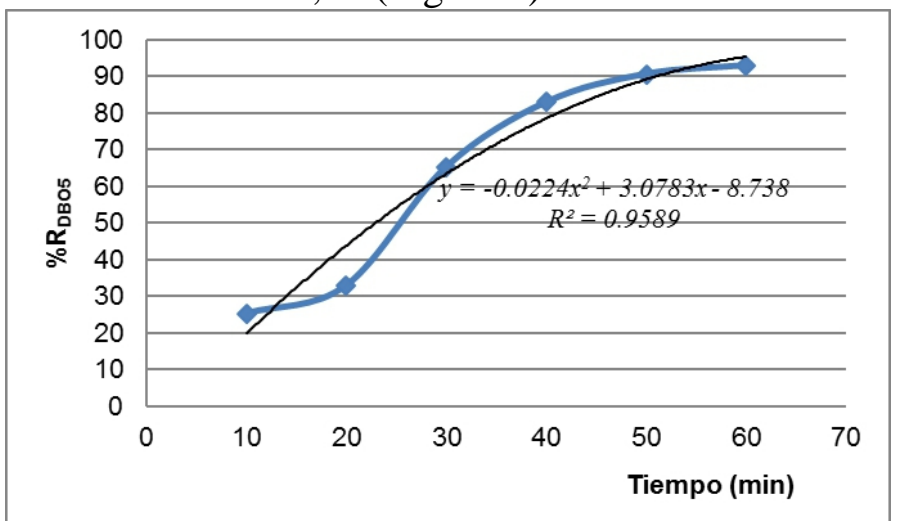

Figura 4. Eficiencia de Remoción de Demanda Bioquímica de Oxígeno

La eficiencia de eliminación de la $\mathrm{DBO}_{5}$ se incrementó según una función polinómica de segundo grado $\left(\mathrm{y}=-0,0224 \mathrm{x}^{2}+3,0783 \mathrm{x}-8,738\right)$, según 
la ecuación de mejor ajuste que se presenta, con coeficiente de correlación $\mathrm{R}$ $=0,98$; se comportó por sobre el $80 \%$ a partir de los 45 minutos de reacción; a partir de este tiempo de oxidación, el proceso no aumentó significativamente, tendiendo al 90\% de eficiencia a partir de los 60 minutos de reacción (Figura 4).

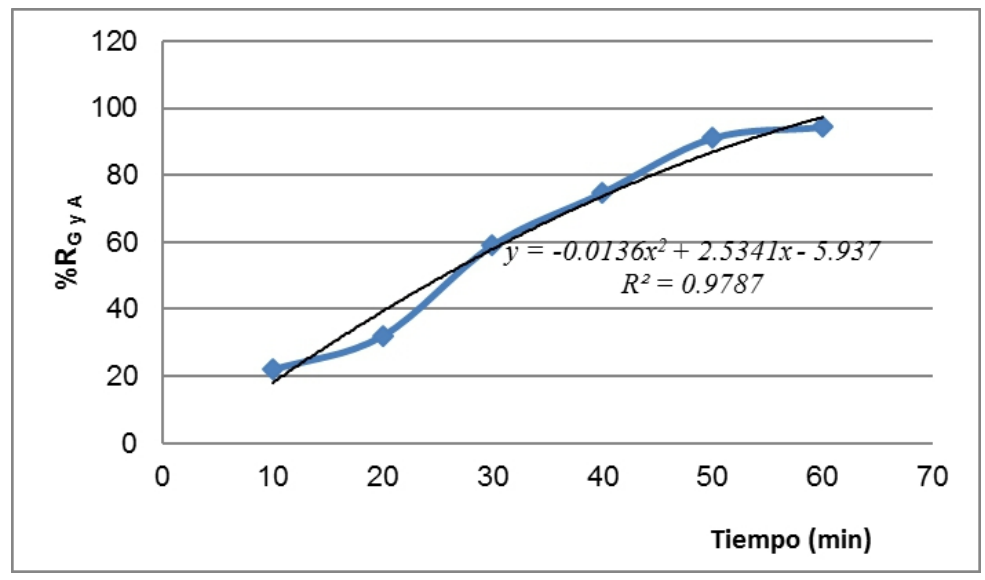

Figura 5. Eficiencia de Remoción de Grasas y Aceites

Por último se estableció el comportamiento de la ozonización de las grasas y aceites contenidos en los residuos líquidos de la industria textil, obteniéndose la mayor eficiencia de remoción de todos los parámetros, en 50 minutos de reacción oxidativa, indicando lo químicamente oxidables que son estos compuestos. El proceso se ajustó a una función de segundo grado ( $\mathrm{y}=-$ $\left.0,0136 x^{2}+2,5341 x-5,937\right)$ con 0,99 de ajuste (Figura 5).

\section{Conclusiones}

Los diferentes parámetros indicadores de la contaminación cuya oxidación por ozonización fueron estudiados, es decir, Color, Turbidez, Sólidos Suspendidos Totales, Demanda Química de Oxígeno, Demanda Bioquímica de Oxígeno y, Grasas y aceites, mostraron niveles oxidativos satisfactorios, siendo la $\mathrm{DBO}_{5}$ y las Grasas y aceites los más oxidados a los 50 minutos de reacción. El otro parámetro que mostró resultados oxidativos satisfactorios fue el color, aunque apreciablemente al cabo de los 60 minutos.

Todos los parámetros indicadores de la contaminación estudiados resultaron oxidados vía ozonización, según comportamientos polinómicos de segundo grado, con coeficientes de correlación por sobre los 0,98 ; lo cual es suficientemente alto como para justificar esta afirmación.

\section{References:}

1. APHA, AWWA, WPCF. 2005. Standard Methods for the Examination of Water and Wastewater. 21th Edition. New York. USA. 
2. Bolaños A. R. A. 2010. Propuesta de recuperación del agua residual proveniente de la industria textil. Tesis presentada en opción al título de Ingeniero Químico. Escuela de Ingeniería Química, Facultad de Ingeniería y Arquitectura. Universidad de El Salvador. El Salvador.

3. De Jesús, T., Rodríguez, H. y Masó, A. 2016. Caracterización de las aguas residuales generadas en una industria textil cubana. Ingeniería Hidráulica, RIHA Vol. 37, No. 3. La Habana, Cuba.

4. Leiva et al. 2016. Estudio preliminar sobre la decoloración del residual líquido de la producción de papel mediante ozonización. European Scientific Journal, February 2016, Vol.12, No.9.

5. Levenspiel, O. 2004. Ingeniería de las reacciones químicas. Ed. Limusa - Wiley, 3ra. Edición. México, D.F. 